\title{
Modularity of the Neck in Birds (Aves)
}

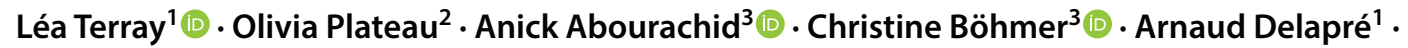 \\ Xavier de la Bernardie ${ }^{4} \cdot$ Raphaël Cornette $^{1}$
}

\begin{abstract}
The neck connects the head and the trunk and is the key structure allowing all movements of the head. The neck morphology of birds is the most variable among living tetrapods, including significant differences in the number and shape of the cervical vertebrae. Despite these differences, according to the literature, three morphofunctional regions (i.e., modules) have been identified along the neck. However, this regionalization has not been quantitatively tested through a geometric morphomet-ric approach applied to the cervical vertebrae. Based on the examination of 187 cervical vertebrae belonging to 16 species with various ecologies, we revealed a common modular structure of the neck using 3D surface geometric morphometrics. We adopted an approach without a priori clustering to identify modules along the neck. The phylogenetic influence on each module was tested. Then, each module was digitally reconstructed as a 3D vertebral model, and postural characteristics were studied. We characterized 9 modules: 7 are transpecific, being shared by at least 2 and up to 15 species. Two modules are specific to species with particularly long necks. The modularity pattern appears to be tightly linked to morphofunctional aspects and partially to phylogeny. In contrast, feeding ecology seems to be more closely related to the chaining of mod-ules (the neck) than to the modules themselves. A study of postural properties revealed that each modular unit exhibits a characteristic curvature. Overall, the modular structure of the neck corresponds to the three traditional functional regions. However, the results also revealed unexpected pattern complexity, including subdivisions within these regions. The study of the patterns of modularity is therefore a relevant approach for challenging the three-functional-region hypothesis and allowed us to identify the structure of the diversity of the necks of birds.
\end{abstract}

Keywords Functional biology $\cdot$ Modularity $\cdot$ Birds $\cdot$ Cervical vertebrae $\cdot$ Geometric morphometrics

Lea Terray and Olivia Plateau have Equal Authorship.

Electronic supplementary material

Léa Terray

lea.terray@mnhn.fr

$\triangle$ Olivia Plateau

olivia.plateau@unifr.ch

1 UMR 7205 Institut de Systématique, Evolution, Biodiversité (ISYEB), Muséum National D'Histoire Naturelle, CNRS, Sorbonne Université, EPHE, Université des Antilles, CP 50, 57 rue Cuvier, 75005 Paris, France

2 Faculté des sciences, Département de géosciences, Université de Fribourg, Chemin du musée 6, 1700 Fribourg, Switzerland

3 UMR 7179 Mécanismes Adaptatifs Et Evolution (Mecadev), Muséum National d'Histoire Naturelle - CNRS., CP 55, 57 rue Cuvier, 75231 Paris, France

4 UMR 6457, SUBATECH, IMT-Atlantique, Université de Nantes, CNRS/IN2P3, 4 rue Alfred Kastler, Nantes, France

\section{Introduction}

The neck, which is morphologically defined as an association of multiple cervical vertebrae (Romer 1950), is a key structure of tetrapods (limbed vertebrates) facilitating varied complex movements (Long et al. 1997; Johnson and Shapiro 1998; Daeschler et al. 2006; Ericsson et al. 2013; Pierce et al. 2013). In contrast to mammals, which almost exclusively exhibit seven cervical vertebrae, irrespective of neck length (Galis 1999), the number of vertebrae in the necks of birds varies greatly (Böhmer et al. 2019), ranging from ten cervical vertebrae in many parrots to 26 cervical vertebrae in the swan (Boas 1929; Böhmer et al. 2019). In addition to this numerical variability, birds display a high shape variability of the cervical vertebrae across species (Boas 1929). The differences in vertebral morphology and number appear to be linked to behavioral adaptations involving special feeding techniques (as observed in the darter bird Anhinga anhinga 
Boas 1929) or peculiar locomotion modes (Dilger 2010; Müller et al. 2010; Wilkinson and Ruxton 2012). This high diversity of shape and associated functions is associated with a wide range of ecologies (Stevens and Parrish 1999; Graf et al. 1994; Alexander 1985); for example bassoon nuts are fishing birds, while woodpeckers are strongly specialized to extract insects directly from tree bark.

Despite the wide variation in the number and morphology of cervical vertebrae, the necks of birds have traditionally been divided into three main morphofunctional regions. Inspired by Virchow (1910), who investigated the mobility of the neck in the penguin, Boas (1929) compared neck mobility across a larger sample of bird species. According to differences in maximal dorsoventral mobility between successive vertebrae, the cervical vertebral column of birds can be divided into three main regions (Virchow 1910; Boas 1929). The cranial portion is characterized by prevalent ventral flexion, the intermediate region is prevalently flexible in the dorsal direction, and the caudal portion allows movements in both directions. Several studies have supported this regionalization of the avian neck (e.g., Zweers et al. 1987; Heidweiller et al. 1992; Bout 1997; van der Leeuw et al. 2001; Tambussi et al. 2012; Cobley et al. 2013; Krings et al. 2017; but see also Kambic et al. (2017), who challenged this three-region model).

The concept of modularity suggests the existence of modules, or sets of traits that show greater covariance within each set than compared with traits of other sets and, thus, evolve partially independently (Olson and Miller 1958; Riedl 1978; Cheverud 1996; Klingenberg 2008, 2010; Hallgrimsson et al. 2009). Three main types of modularity exist (West-Eberhart 2003; Klingenberg 2008; Hallgrimsson et al. 2009): developmental, functional and evolutionary modularity, which affect morphological variation at different scales. Modules are generally defined based on shape covariation (Klingenberg 2008). Here, because we worked on serially homologous structures (vertebrae), we assessed degree of shape covariation between them based on their morphological proximity. We define a module as a set of homologous structures that are the most similar. The patterning and regionalization of the vertebral column is a topic that has been well studied in a broad panel of tetrapods (e.g., Polly et al. 2001; Hautier et al. 2010; Asher et al. 2011; Arnold et al. 2016; Randau et al. 2017 among others); however, within birds, such studies have been quite limited (e.g., Guinard et al. 2010; Guinard and Marchand 2010; Guinard 2012; Azevedo et al. 2012; Böhmer et al. 2015). Diverse methods have been used to quantify these characteristics, such as traditional linear distance approaches (e.g., Guinard et al. 2010; Guinard and Marchand 2010), among which geometric morphometrics (GM) is the most widespread method for the study of morphological modularity (Klingenberg 2008; Klingenberg and Marugan-Lobon
2013; Klingenberg 2014; e.g., Böhmer et al. 2015; Head and Polly 2015; Randau et al. 2017). However, previous studies on the modularity of the neck of birds have focused only on a single taxon or a few taxa have often applied a developmental perspective to the structure. At present, a large-scale analysis of the modularity of the necks of birds using stateof-the-art statistical shape methods is lacking.

The aim of this study was to reveal how shape diversity is structured in bird necks by studying the patterns of modularity at the interspecific level. To this end, we investigated the complete cervical vertebral columns of 16 different bird species with varied ecologies using a three-dimensional surface GM (3D SGM) approach, allowing us to accurately quantify the entire shape of the cervical vertebrae. We chose an innovative approach to investigate modularity, and we defined a module as a set of vertebrae that share a common morphology and are more similar to each other compared to the vertebrae outside the module. This definition is applicable because vertebrae are serially homologous structures. To identify modules, vertebrae were grouped using an approach without a priori clustering. For each module, the phylogenetic signal was assessed, and postural properties in a relaxed posture were studied. We discuss the relative importance of three potential factors: feeding ecology, phylogeny, and morphofunctional aspects, at two different scales of shape variation: the modules and the neck, and we then challenge the three commonly established functional regions in light of our results.

\section{Material and Methods}

\section{Sample}

We sampled the cervical vertebral columns of 16 extant bird species (Table 1). The atlas (first cervical vertebra) was not included in the analysis due to its unique morphology and the lack of specific anatomical homologies with the postatlantal cervical vertebrae. In total, 3D models of 187 cervical vertebrae (CVs) were obtained. The taxa were chosen to be distributed within the phylogenetic tree and to provide a wide range of body sizes (from the $7.5 \mathrm{~cm}$ colibri to the $2.5 \mathrm{~m}$ ostrich) and feeding ecologies (piscivores, frugivores, granivores, nectarivores, scavengers) within the limits of the available specimens in the Muséum National d'Histoire Naturelle osteological collection.

\section{D scans}

3D models of the specimens were generated using different scanning methods depending upon the size of the bones to achieve the best results. The CVs of the four largest specimens in the sample were digitized using Breuckmann 
Table 1 Sampling

\begin{tabular}{|c|c|c|c|c|c|}
\hline$\overline{\text { Genus }}$ & Species & $\begin{array}{l}\text { Number of } \\
\mathrm{CVs}\end{array}$ & Collection number & Missing data & Feeding ecology \\
\hline Crypturellus & cinereus & 16 & MNHN 2004-187 & - & $\begin{array}{l}\text { Insectivorous } \\
\text { Frugivorous } \\
\text { Granivorous }\end{array}$ \\
\hline Apteryx & $s p$. & 14 & BMNH 17-01-72-1 & - & Insectivorous \\
\hline Struthio & camelus & 18 & MNHN 1908-160 & - & $\begin{array}{l}\text { Insectivorous } \\
\text { Frugivorous } \\
\text { Granivorous }\end{array}$ \\
\hline Chlorostilbon & mellisugus & 11 & MNHN 2000-466 & CV 6 & Nectarivorous \\
\hline Gallirallus & gallirallus & 13 & MNHN 1870-182 & - & Insectivorous \\
\hline Aptenodytes & patagonicus & 12 & Unumbered & - & Piscivorous \\
\hline Anhinga & anhinga & 18 & MNHN 1885-543 & CV 10 & Piscivorous \\
\hline Morus & bassanus & 15 & MNHN 1997-189 & - & Piscivorous \\
\hline Gyps & fulvus & 15 & MNHN 1996-43 & - & Scavenger \\
\hline Dryocopus & martius & 11 & MNHN 2013-344 & CVs $3,4,8$ & Insectivorous \\
\hline Cacatua & moluccensis & 11 & MNHN 2000-88 & CV 2, 8 & Frugivorous \\
\hline Amazona & dufresniana & 12 & MNHN 2004-198 & - & Frugivorous \\
\hline Calyptomena & viridis & 11 & MNHN 1997-886 & CV 2, 11 & $\begin{array}{l}\text { Frugivorous } \\
\text { Granivorous }\end{array}$ \\
\hline Oriolus & oriolus & 12 & MNHN 1993-131 & - & $\begin{array}{l}\text { Frugivorous } \\
\text { Granivorous }\end{array}$ \\
\hline Bombycilla & garullus & 12 & MNHN 2010-115 & CV 9 & $\begin{array}{l}\text { Frugivorous } \\
\text { Granivorous }\end{array}$ \\
\hline Hirundo & rustica & 13 & MNHN 2000-733 & CV 3 & Granivorous \\
\hline
\end{tabular}

$B M N H$ British Museum of Natural History, MNHN Muséum National d'Histoire Naturelle Paris

3D surface scanners at the Museum National d'Histoire Naturelle, Paris (UMS 2700): the taxa of intermediate size (Aptenodytes, Morus, Gyps) were digitized using a bluelight fringe 3D scanner (SmartScan 3D model) and the larger taxon Struthio using a white-light fringe 3D scanner (StereoScan 3D model with a camera resolution of 5 megapixels). Then, Geomagic (Geomagic Studio; Raindrop Geomagic, Research Triangle Park, North Carolina, USA) was used to prepare the scans as described by Botton-Divet et al. (2015). The other 12 specimens were scanned with an RX Solutions microtomograph (Easy Tom 4) with the following settings::U $=100 \mathrm{kV}, \mathrm{I}=[100-386] \mu \mathrm{A}$, helical trajectory, voxel size $=11.3 \mu \mathrm{m}$ for Hirondo and Chlorostilbon, $23.3 \mu \mathrm{m}$ for Oriolus and Bombycilla and $54.3 \mu \mathrm{m}$ for other genera. The reconstruction software was RX Solutions XAct $2.0 \mathrm{r}$ 8177. The dataset was then segmented, and surface meshes were generated using Avizo software.

\section{D Surface Geometric Morphometrics}

Vertebrae exhibit a particularly complex shape that cannot be precisely recorded through traditional landmark-based procedures. Therefore, we applied a 3D surface sliding landmark approach (Bookstein 1997; Gunz et al. 2005) using three types of landmarks: homologous anatomical landmarks and sliding semilandmarks of curves and surfaces.
The anatomical landmarks and sliding semilandmarks of curves were manually digitized using the Landmark software (Wiley et al. 2005), and the sliding semilandmarks of surfaces were positioned using sliding landmarks and relaxation procedures. This was done following the protocol of Gunz et al. (2005), and the workflow is detailed by Botton-Divet et al. (2015). The sliding landmarks procedure was performed using the placePatch function of the Morpho package (Schlager 2013) in the free software R ( $R$ Core team 2017). We first designed a template following the method of Cornette et al. (2013). Second, the function placePatch automatically projected the sliding semilandmarks of surfaces from the template on all other scans via thin-plate spline deformation. To be more accurate, the template was deformed to correspond to the anatomical landmarks and sliding semilandmarks of the curves of the target specimen, and the coordinates of the sliding semilandmarks of the surfaces of the deformed template were then projected on the target specimen. Subsequently, relaxation against the template was performed using the relaxLM function from the same package, allowing sliding semilandmarks of surfaces to freely slide along the surface to minimize bending energy. The sliding and relaxation procedures were both repeated iteratively. Thereafter, to remove biases linked to the first relaxation against the template, a second spline relaxation procedure was executed against the mean of all objects 
with the slider3d function from the same package. Sliding semilandmarks are geometric homologs, and because of these procedures, all landmarks of the dataset could be compared and analyzed using traditional morphometrics. In total, 16 anatomical landmarks, 651 sliding semilandmarks of curves and 159 sliding semilandmarks on surfaces were used (Fig. 1, Table 2).

All landmark coordinates were superimposed via generalized Procrustes analysis (GPA) to remove the nonshape effects of rotation, translation and scale (Rohlf and Slice 1990) using the gpagen function of the Geomorph package (Adams and Otárola-Castillo 2013).

All subsequent statistical treatments and analyses were also performed using the free software R ( R Core team 2017).

\section{Module Identification}

We define a module as a set of CVs that share similar morphologies. To identify and characterize these modules, we used an unsupervised pattern recognition method: Gaussian mixture analysis (Fraley and Raftery 1998; Everitt and Dunn 2001). Gaussian mixture analysis is a classification method that identifies Gaussian subsets within the main Gaussian of the dataset without prior information on the objects. The Gaussian assumption is especially adapted to morphometric data. First, size elimination and projection into reduced space, as performed by principal component analysis (PCA), contribute to the normalization of the data
(Diaconis and Freedman 1984). Then, unsupervised clustering is performed, which is a highly efficient method for datasets whose sizes and shapes may vary between groups (Baylac et al. 2003; Cordeiro-Estrela et al. 2006), as is the case for morphometric data.

We also performed PCA of the Procrustes coordinates using the plotTangentSpace function of the Geomorph package (Adams and Otárola-Castillo 2013). We retained 90\% of the shape variability as shape variables. Only $90 \%$ of the variability was considered because the last principal components are generally considered noise (Baylac and Frieß 2005). For these variables, we performed a model-based clustering analysis, applying the Mclust function from the mclust library (Fraley and Raftery 2012). This function finds the number of clusters without a priori, according to the covariance structure of the dataset. It is based on an algorithm designed by Dasgupta and Raftery (1998), and generalized by Fraley and Raftery (1998). Here are the main steps of the algorithm, as written by Fraley and Raftery (1998): "(1) Determine a maximum number of clusters to consider (M) and a set of candidate parametrizations of the Gaussian model to consider. In general $\mathrm{M}$ should be as small as possible. (2) Do agglomerative hierarchical clustering for the unconstrained Gaussian model, and obtain the corresponding classifications for up to M groups. (3) Do EM (Expectation Maximization algorithm) for each parametrization and each number of clusters [from 2 to $\mathrm{M}$ ] starting with the classification from hierarchical clustering. (4) Compute the BIC (Bayesian Information Criterion) for the one-cluster
Fig. 1 Cervical vertebrae anatomy and landmarks locations used in our analysis (sixth vertebrae of Gyps fulvus). Red points: 16 anatomical landmarks; blue points: 651 sliding semilandmarks of curves; green points: 159 sliding semilandmarks of surfaces (Color figure online)

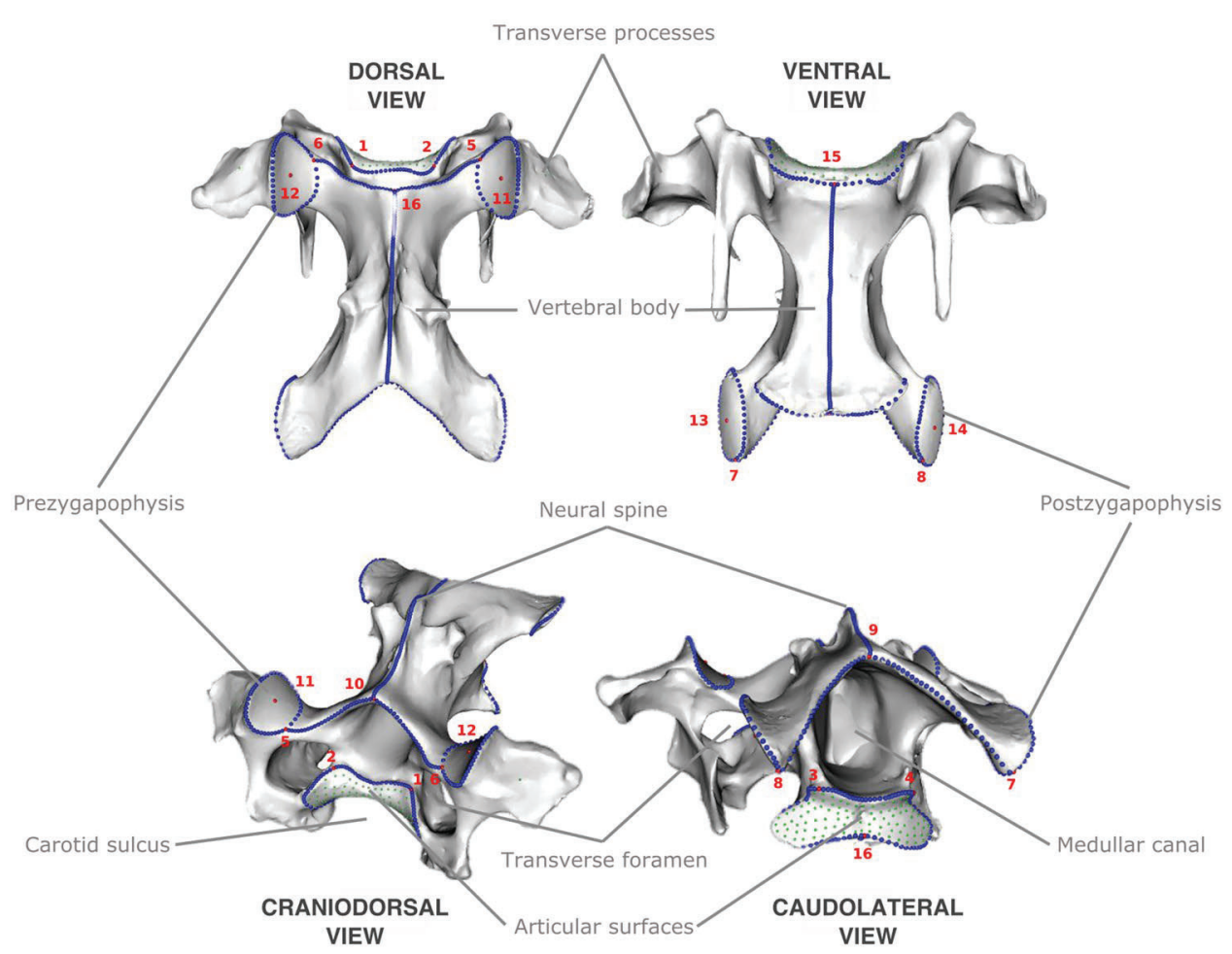


Table 2 Definition of the anatomical landmarks, semilandmarks of curves (define between anatomical landmarks) and semilandmarks of surfaces (define by semilandmarks of curves)

\begin{tabular}{ll}
\hline & Definitions \\
\hline Anatomical Landmarks & \\
1 & Maximum curvature of the dorsal and right part of the cranial articular surface \\
2 & Maximum curvature of the dorsal and left part of the cranial articular surface \\
3 & Maximum curvature of the dorsal and left part of the caudal articular surface \\
4 & Maximum curvature of the dorsal and right part of the caudal articular surface \\
5 & Junction of vertebral arch and left prezygapophyse facet \\
6 & Junction of vertebral arch and right prezygapophyse facet \\
7 & Junction of vertebral arch and right postzygapophyse facet \\
8 & Junction of vertebral arch and left postzygapophyse facet \\
9 & Caudal maximum curvature of vertebral arch \\
10 & Cranial maximum curvature of vertebral arch \\
11 & Middle of left prezygapophyse facet \\
12 & Middle of right prezygapophyse facet \\
13 & Middle of right postzygapophyse facet \\
14 & Middle of left postzygapophyse facet \\
15 & Middle ventral curvature of the cranial articular surface \\
16 & Middle ventral curvature of the caudal articular surface \\
Semilandmarks of curves & \\
$1-2-15$ & Outline of the cranial articular surface \\
$3-4-16$ & Outline of the caudal articular surface \\
$5-10-6$ & Border of the cranial face of the vertebral arch \\
$8-9-7$ & Border of the caudal face of the vertebral arch \\
5 & Outline of left prezygapophyse facet \\
6 & Outline of right prezygapophyse facet \\
7 & Outline of left postzygapophyse facet \\
$9-10$ & Outline of right postzygapophyse facet \\
$15-16$ & Central line along the dorsal face of the vertebral arch \\
$3-4-16$ & \\
\hline & \\
\hline &
\end{tabular}

model for each parametrization and for the mixture likelihood with the optimal parameters from EM for [2 to M] clusters. This gives a matrix of BIC values corresponding to each possible combination of parametrization and number of clusters. (5) Plot the BIC values for each model. A decisive first local maximum indicates strong evidence for a model (parametrization + number of clusters)." In this study, the VEI model (model: $\lambda \mathrm{kA}$, distribution: diagonal, volume: variable, Shape: equal, orientation: coordinate axes) (Fraley and Raftery 2012) was used to parameterize the covariance matrix.

To quantify the significance of those modules, we tested the reciprocal influence of modules and species on the overall vertebrae shape. We performed a Procrustes MANOVA with permutation procedures, a resampling method that aim to validate the model by using random subsets of the data. We used the function 'procD.lm' from package 'geomorph' (Adams and Otárola-Castillo 2013). Then the proportion of variance that correspond to each of these factors was calculated according to the formula: $\mathrm{SSf} / \mathrm{SSt}=\mathrm{V}$, where SSf is the sum of squares for the factor, SSt the total sum of squares and V the proportion of variance explained by the factor.

For each identified subset (i.e., module), we constructed 3D mean shape meshes. First, mean landmark coordinates were calculated using the mshape function of the Geomorph package (Adams and Otárola-Castillo 2013). Then, a thin plate spline deformation was applied on the closest mesh from the mean shape of the module (identified using the findMeanSpec function), so that the deformed mesh corresponded to the mean landmark coordinates calculated with the warpRefMesh function. Each mean mesh was exported using the mesh2ply function. 


\section{Shape Trajectories}

To obtain a better overview of the diversity of the neck, we plotted the shape trajectories of each species using the ggplot and geom_segment functions from the ggplot2 package (Wickham 2015). Shape trajectories are the trajectories of shape changes between successive CVs in the PC1 vs. PC2 morphospace (raw PCAs with shape trajectories are available in SP Fig. 2) (i.e., the morphospace trajectory from the first to the last $\mathrm{CV}$ ). This approach provides a simple visual representation of shape changes along the neck, as explained by Werneburg et al. (2015). We then visualized the order of the modules along the neck using the mean shape meshes of Fig. 2.

\section{Influence of Phylogeny on the Modules}

The phylogenetic influence on each module was tested, and the results are presented in Table 2. The hypothesis regarding the phylogenetic relationships of the birds considered in our study was based on molecular data (Hackett et al. 2008). A consensus topology (strict consensus tree) was generated from downloaded samples (100 randomly selected phylogenetic trees from the Global Bird Tree (Jetz et al. 2012) using the phytools, ape, picante and geiger packages in $\mathrm{R}$ (Paradis et al. 2004; Harmon et al. 2008; Kembel et al. 2010; Revell 2012). To quantify the phylogenetic influence, we used the K-statistic method developed by Blomberg et al. (2003) and adapted to multivariate data by Adams (2014), which quantifies the phylogenetic signal within datasets containing several variables such as shape. The phylogenetic signal was assessed for each module independently except for module 7 , module 8 and module 9 , for which the signal was not computable because these modules were present only in one or two species. A high $\mathrm{K}$-value indicates a strong phylogenetic signal, meaning that the corresponding module is influenced by strong phylogenetic constraints. K-values were calculated using the physignal function in the Geomorph library (Adams and Otárola-Castillo 2013).

\section{Reconstruction of the Osteological Neutral Posture for Each Module}

To explore the effect of the specific morphology of each module on its curvature in the osteological neutral posture (Stevens and Parrish 1999), we reconstructed global mean modules and measured their curvature angle, both between two CVs and for the entire module. The reconstructions of the modules were generated by duplicating the mean shape meshes a number of times, where the number of duplications was equivalent to the number of $\mathrm{CV}$ s per module, which was estimated from an average calculation based on our sample. Using the software blender ver. 2.79 (Blender Foundation
2003-2018), we assembled the duplicated CVs in anatomical connection so that the articular surfaces of the vertebral body and the pre- and postzygapophyses of two successive CVs were in contact with each other without overhang. This arrangement based on osteological criteria does not take into account additional constraints/possibilities due to soft tissue such as muscles and ligaments.

\section{Results}

\section{Module Identification}

We identified nine statistically significant modules. Seven of them were found in the studied species (SP Fig. 1).

The manova testing the influence of modules and species revealed that both factors significantly influence vertebrae shape (modules: p-value $<0.05$; species: p-value $<0.05$ ). Modules explain $63 \%$ of vertebrae shape, while species explain $13 \%$.

The comparison of the mean shape of each module presented in Fig. 2 reveals that the modules are characterized by specific morphological features. Transversal processes appear to be one of the less variable features from one module to another.

\section{Module 1}

The CVs of the first module are characterized by a welldeveloped neural spine that points vertically. They exhibit rather large, ventrally curved prezygapophyses and slightly ventrally inclined postzygapophyses. Another characteristic feature is the ventral process on the centrum. In dorsal view, the CVs present an X-shape.

\section{Module 2}

The characteristic features of the CVs in this module include the presence of well-developed transverse processes, including fused cervical ribs and a mostly enclosed carotid sulcus. In cranial view, the articular surface of the prezygapophyses is inclined medially. The orientation of the articular surface of the postzygapophyses is slightly ventrally inclined in lateral view. The neural spine is rather low.

\section{Module 3}

The CVs are compact with a high and wide vertebral body. In cranial view, the articular surface of the prezygapophyses exhibits a dorsomedial orientation. They are less concave than those of the second module. The articular surface of the postzygapophyses is rather horizontal in lateral view. The 
Fig. 2 Mean shape meshes of the nine modules identified by Gaussian mixture
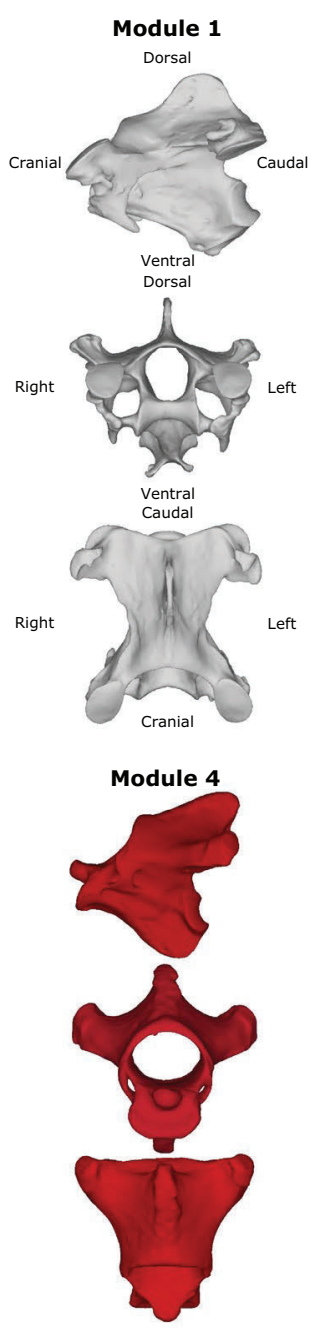

Module 7
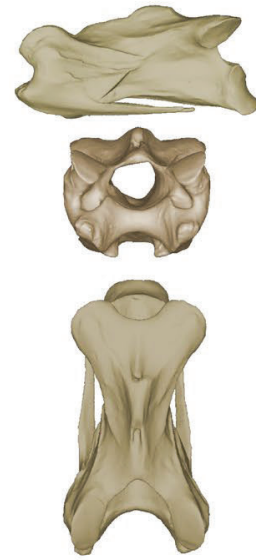
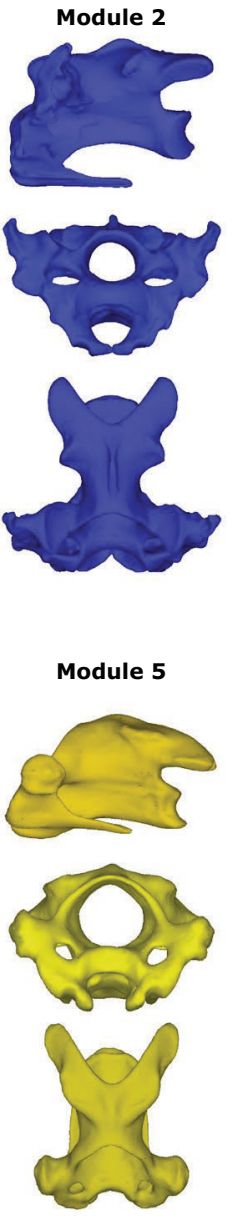

Module 8

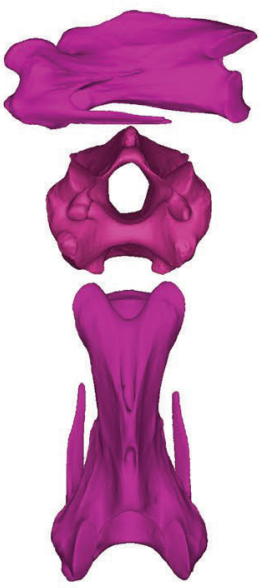

Module 3
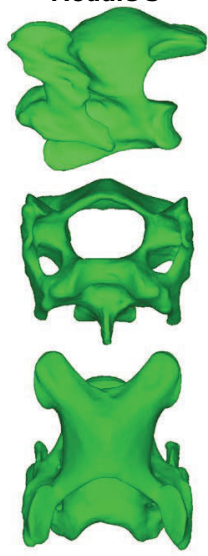

Module 6
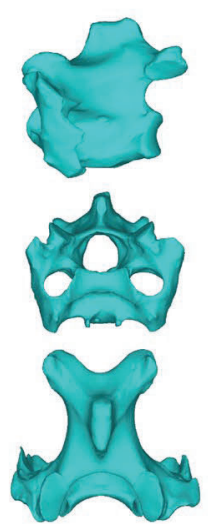

Module 9

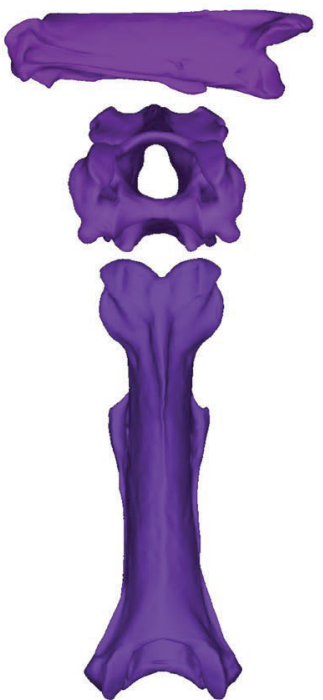


medullar canal is oval, horizontally elongated, and hypapophysis is well developed.

\section{Module 4}

The fourth module comprises only one $\mathrm{CV}$, which is the axis (the second cervical vertebra). Compared to the postaxis CVs in the neck, it is very peculiar in its morphology and consequently forms a group of its own. The most pronounced feature is the absence of a prezygapophysis and the presence of the odontoid process cranially articulating with the atlas (the first $\mathrm{CV}$ ) and the occiput of the skull. In lateral view, the well-developed neural spine projects caudally, with the caudal border being almost aligned with the postzygapophyses. The axis presents a well-developed ventral process. Transverse foramina are missing. The postzygapophyses are ventrally oriented.

\section{Module 5}

The morphology of the CVs of module 5 resembles that of module 2, but the neural arch has a rounded shape in lateral view, and the spinal canal is rather large. The articular surface of the prezygapophyses has a dorsomediocaudal orientation; that of the postzygapophyses has a ventrolateral orientation. The carotid sulcus is marked.

\section{Module 6}

The CVs of this module are compact (similar to module 3) with well-developed neural spines. The articular surface of the prezygapophyses has a dorsomedial orientation in cranial view. The articular surface of the postzygapophyses presents a ventrolateral orientation. The spinal canal is rounded, and the carotid sulcus is slightly marked.

\section{Module 7}

The CVs are elongated and characterized by a rather small neural spine. The articular surface of the prezygapophysis is inclined dorsomedially, whereas the articular surface of the postzygapophyses are inclined ventrolaterally. The postzygapophyses are very short and are set back from the caudal end of the vertebral centrum. The carotid sulcus is marked. The transverse processes develop with long fused cervical ribs. This module was only identified in the neck of Anhinga and Struthio specimens.

\section{Module 8}

The CVs of module 8 also display an elongated morphology, even more so than those of module 7. The articular surface of the prezygapophyses is inclined ventrally in lateral view and medially in cranial view. The articular surface of the postzygapophyses is inclined ventrally. The carotid sulcus is marked. The transverse processes develop with long fused cervical ribs, which are shorter than those of module 7 . The medullar canal is oval, being vertically elongated. This module was only identified in the neck of Struthio specimens.

\section{Module 9}

The CVs of module 9 are extremely elongated, more so than those of modules 7 and 8 . In cranial and lateral view, the articular surface of the pre- and postzygapophyses are inclined ventrally. In particular, the postzygapophyses are very long and project caudally, extending over the caudal end of the vertebral centrum. The carotid sulcus is marked. This module was only found in the neck of Anhinga specimens.

\section{Shape Trajectories}

Species' shape trajectories-i.e., morphospace trajectory from the second to the last $\mathrm{CV}$ - are presented in Fig. 3.

All modules are present in more than one species except for modules 8 and 9, which are only present in Anhinga. The number of CVs constituting each module is quite stable; however, it is different between the modules. For instance, module 1 is generally composed of two or three CVs, and module 4 is always composed of one CV. The distribution of the modules along the neck is quite similar among species. For example, module 4 contains only the second cervical vertebra of each species, and module 1 is always found subsequent to module 4 . Some other modules occupy the same place in the shape trajectory as modules 2 and 5 .

The shape trajectories can be divided into two categories based on the shape of the trajectory. The first category includes trajectories that display a reversed C-shape (Calyptomena, Bombycilla, Amazona, Dryocopus, Aptenodytes, Morus, Oriolus and Cacatua). In some members of this group, the first and last vertebrae of the shape trajectory are truly close to each other in the morphospace. This is observed for Aptenodytes, Dryocopus and Morus (Fig. 3). In addition, Morus shows some peculiarities regarding the positions of the CVs of module 2 (Fig. 3). The second category of shape trajectories has a crushed reversed C-shape, meaning that the lower arc of the shape trajectories rises against the upper arc (Chlorostilbon, Crypturellus, Gyps, Apteryx and Hirundo). Among the members of this group, Chlorostilbon and Apteryx show peculiar shape trajectories (Fig. 3). Two species could not be assigned to one of these two general categories: Anhinga and Struthio. 


\section{Reconstruction of the Osteological Neutral Posture for Each Module}

The modules are variable in the number of CVs and result in different curvatures in an osteological neutral posture (Fig. 4). Module 4 was not subjected to this treatment because it is always composed of only one CV. There are three observable classes of modules: those that exhibit ventral curvature, such as modules 1 and $9\left(21.09^{\circ}\right.$ to $21.44^{\circ}$ of curvature, respectively, between two CVs); those with dorsal curvature, such as modules 2 and 5 (16.32 ${ }^{\circ}$ and $19.37^{\circ}$, respectively); and one that show very low curvature or does not show any as module $3,6,7$ and 8 (from $0^{\circ}$ to $6.47^{\circ}$ ). We observed that modules that share common postural properties in a relaxed posture occupy the same place in the neck. Modules in the ventral curvature belong to the
Fig. 4 Reconstruction of eight of the nine mean modules. Module 4 was not reconstructed because it was always composed of one vertebra. Curvature angles have been measured between two consecutive vertebrae and between the first and the last (i.e. total curvature angle of the module in relaxed posture)
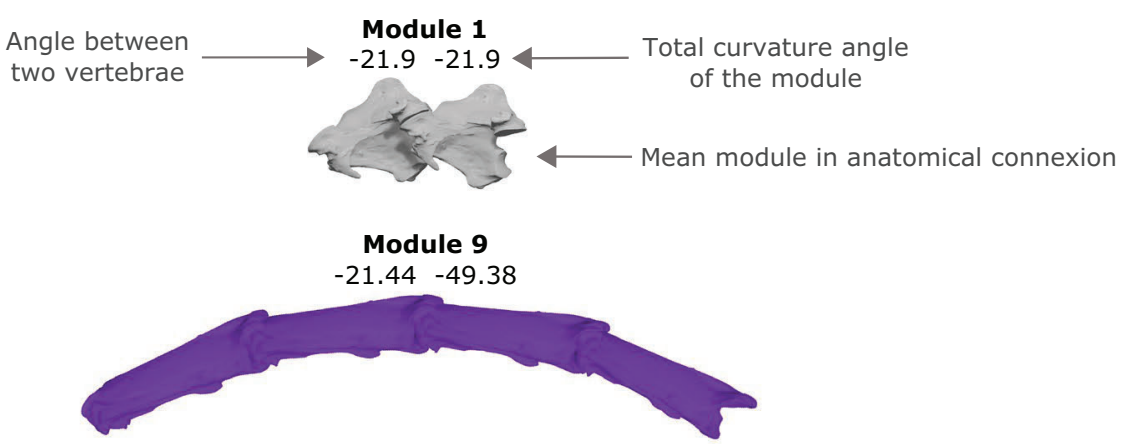

Cranial morpho-functional region

Intermediate morpho-functional region

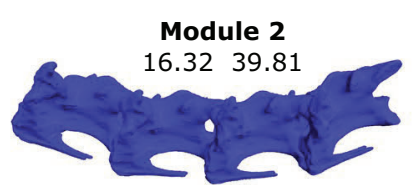

Module 5

19.3726 .86

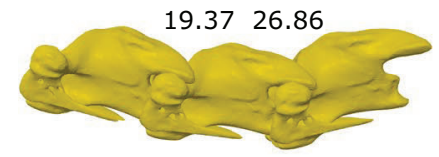

Caudal morpho-functional region

Module 3

$6.47 \quad 12.61$

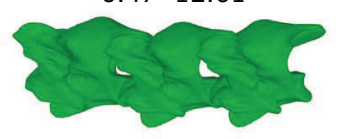

Module 6

00

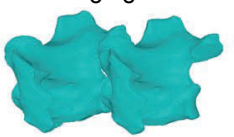

Module 7

3.846 .09

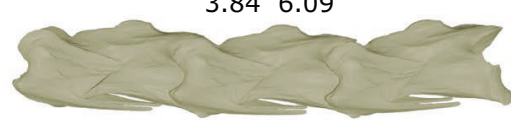

Module 8

2.537 .69

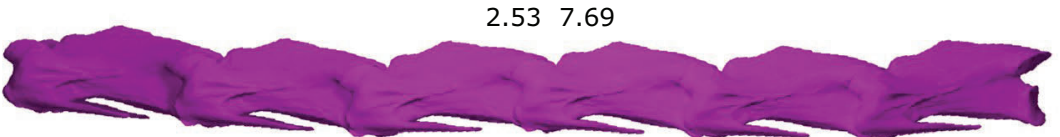


cranial morphofunctional region, those with dorsal curvature belong to the intermediate region, and those with little or no curvature belong to the caudal region.

\section{Discussion}

To investigate modularity in the necks of birds, we applied a 3D SGM approach to the CVs of 16 species with varied feeding ecologies. We statistically identified nine modules. Seven of the modules are transpecific, while one is specific to Anhinga and one to Struthio, which are two genera with a long, peculiar neck morphology. First, we will discuss the observed morphological diversity at two different scales: modules (i.e., subsets of CVs) and combinations of modules (i.e., necks), and how it may be linked to feeding ecology, phylogeny and morphofunctional aspects. Then, we will discuss the three traditional functional regions of Boas in light of our results.

\section{Modules (i.e., Subsets of CVs)}

The modules are composed of CVs that occupy the same region of the neck (Fig. 3). Depending on their anatomical region, they display peculiar postural properties (Fig. 4). Thus, CVs are distributed in modules according to their postural characteristics, and not according to species. Vertebral shape itself is more explained by the transpecific modules $(63 \%)$ than by species $(13 \%)$. Therefore, modularity pattern along the neck is strongly linked to morphofunctional aspects. This finding supports the hypothesis that vertebral morphology and bending ability are tightly linked to posture and locomotion in vertebrates, as shown in cetaceans (Long et al. 1997), primates (Johnson and Shapiro 1998; Shapiro and Kemp 2019) and xenarthrans (Oliver et al. 2016).

The link between phylogeny and modularity patterns is more subtle. Some modules are under phylogenetic influence, while others are not (Table 3). Thus, phylogeny explains part of the shape variation between modules but does not fully explain the shape variation along the neck. This is in line with the conclusions of Brocklehurst et al. (2018), who postulated that phylogeny applies differentially along the vertebral column. To strengthen these findings, more species must be added to our dataset to further discuss the role of phylogeny in the regionalization of the neck.

The link between feeding ecology and the modularity pattern is less well supported. The modules are transversal across species (Fig. 3, SD Fig. 2), and considering that species represent feeding ecologies, we can deduce that the modular structure of the neck is not mainly linked to feeding ecology. Similar observations have been made in mammals, in which the patterns of modularity in the neck are highly conserved regardless of the species ecology (Arnold et al.
2016, 2017; Villamil 2018), in contrast to those observed in the rest of the column (Jones et al. 2018, 2019). To further extend these conclusions, they can be discussed in light of those reported for Felidae by Randau et al. (2017), who concluded that "ecological factors influence the shape of the vertebral column heterogeneously and that distinct vertebral sections may be under different selection pressures". We can therefore hypothesize that ecological factors might apply heterogeneously along the vertebral column, according to phylogeny. To test this hypothesis, it would be interesting to expand our dataset with other species to obtain statistically testable ecological groups. On the other hand, we can assume that because feeding ecology is not strongly linked to the modularity pattern, it could be linked to the remaining shape variability that exists within modules (SD Fig. 1).

\section{Combinations of Modules (i.e., Necks)}

Great shape variability is also observed at the scale of the neck. This diversity is represented by shape trajectories (Fig. 3), which visually describe morphological variations between CVs along the neck. Similar shape trajectories mean that neck morphologies are linked to similar phylogenetic, developmental and/or environmental factors (Werneburg et al. 2015) and vice versa. We identified two main categories of shape trajectories: reversed $\mathrm{C}$ shape and crushed reversed C shape (Fig. 3). The first group was composed of Calyptomena, Bombycilla, Amazona, Dryocopus, Aptenodytes, Morus, Oriolus and Cacatua and the second of Crypturellus, Gyps, Apteryx, Hirundo and Chlorostilbon, among which the last genus displays a particular shape trajectory. Struthio and Anhinga exhibit very specific shape trajectories and do not belong to one of these groups. These peculiarities may be linked to the particular elongated shape of their neck. Regarding feeding ecologies, the reversed C shape group contains opportunist and species that feed in water (divers and piscivores), while the crushed reversed C shape group contains opportunists, scavengers and nectarivores, among which the last group displays a peculiar trajectory. Therefore, the shape trajectory seems to be linked to feeding ecology. Modules may not appear to be related to ecology, but the chaining of modules (i.e., the neck) seems to be.

\section{The Three Functional Regions Hypothesis}

Each of the nine modules occupies a precise place in the species necks. Apart from module 1, the repartitioning of modules along the neck can generally be divided into three regions: (1) the cranial region, represented by modules 1 and 9 (Fig. 3), resulting in a ventrally bent neutral posture (Fig. 4); (2) the intermediate region, represented by modules 2 and 5 (Fig. 3), resulting in a dorsally bent neutral posture 
(Fig. 4); and (3) the caudal region, represented by modules 3, 6, 7 and 8 (Fig. 3), resulting in a relatively unbent neutral posture (Fig. 4). Together, the ventrally bent cranial region, the dorsally bent intermediate region and the rather unbent caudal region of the cervical vertebral column appear to form the S-shaped neck of birds. These functional descriptions of the three regions of the neck in birds correspond to the observations made by Virchow (1910) and Boas (1929).

However, even though these three regions seem to present a clear functional definition, our results show that there is also a greater diversity than expected: we identified more modules than the regions (nine versus three). This implies the existence of (1) different modules occupying one same functional region and presenting similar postural properties and/or (2) subdivisions within Boas's functional regions (Fig. 3 and Fig. 4):

(1) Modules 2 and 5 occupy the intermediate region and result in a natural dorsally bent posture of approximately $30^{\circ}$. Modules 3, 6, 7 and 8 occupy the caudal region and result in a rather unbent posture. These modules are redundant in terms of their position in the neck and posture. However, they also display significantly different morphologies. The CVs of module 2 exhibit well-developed transverse processes, including fused ribs and a mostly enclosed carotid sulcus. This is in contrast with the CVs of module 5, which display the opposite morphological features (no fused cervical ribs and an open carotid sulcus) (Fig. 2). The same applies to modules 3, 6, 7 and 8, which display progressive elongation along the cranio-caudal axis.

(2) Two redundant modules are commonly found in the same neck, as observed for modules 2 and 5 in the neck of Calyptomena or module 3 and module 6 in the neck of Amazona, Aptenodytes, Chlorostilbon and Crypturellus. Therefore, in some species, the functional regions are morphologically subdivided. These subdivisions consist only of redundant modules and therefore share similar (even if not identical) postural properties but present varied shapes. This finding is in line with those of Krings et al. (2014, 2017), who identified three to seven modules along the neck of owls based on morphological and functional studies.

In conclusion, our results are consistent with those of previous studies on the regionalization of the neck in birds (e.g., Virchow 1910; Boas 1929; Zweers et al. 1987; van der Leeuw et al. 2001; Tambussi et al. 2012; Cobley et al. 2013; Krings et al. 2017; Kambic et al. 2017). However, they indicate that the pattern of modularity is more complex than expected, including subdivisions of the traditional functional regions and module redundancy. We found a clear link between the modular regionalization and posture of the neck. This relationship has been observed in other tetrapod groups (Polly et al. 2001; Hautier et al. 2010; Asher et al. 2011; Arnold et al. 2016; Randau et al. 2017; Jones et al. 2019). The patterns of regionalization in these groups are different, but they display similar pattern complexity.

\section{Conclusion}

In conclusion, despite the great variability of birds in terms of body size, ecology and the number and shape of CVs, the modularity pattern of the neck appears to be shared between species. Our innovative approach for addressing modularity using clustering proved to be relevant for the vertebral column, and nine modules were identified. Seven were transpecific, while one was specific to Anhinga and one to Struthio. The neck of each bird is composed of at least four modules. This modularity pattern is tightly linked to morphofunctional aspects, a property shared with other vertebrate groups, but is also partially linked to phylogeny, which is applied heterogeneously along the column. Feeding ecology seems to be more closely related to the neck than to the modules themselves. Each module results in different naturally bent postures, replicating the characteristic $\mathrm{S}$-shape of the neck of bird. These postural properties of modules along the neck correspond to Boas's (1929) functional regions. However, we also found a more complex pattern than expected: first, these regions are subdivided; then, one region can be occupied by different modules with same postural properties between one species and another.

Acknowledgements The authors thank Pr Christine Lefèvre for the access of specimens from the collections (Muséum National d'Histoire Naturelle, Paris). They also thank Amandine Blin from the 'plate-forme de morphométrie' of the UMS 2700 (CNRS, MNHN) for access to the surface scanner.

Funding This study was financially supported by the Action transversale du Muséum (ATM) and the Agence National de la Recherche (ANR): Project ID \#ANR-16-CE33-0025 (AVINECK), Project Coordinator: A. Abourachid.

\section{Compliance with Ethical Standards}

Conflict of interest The authors declare that they have no conflict of interest.

\section{References}

Adams, D. C. (2014). A generalized K statistic for estimating phylogenetic signal from shape and other high-dimensional multivariate data. Systematic Biology, 63(5), 685-697.

Adams, D. C., \& Otárola-Castillo, E. (2013). Geomorph: An r package for the collection and analysis of geometric morphometric shape data. Methods in Ecology and Evolution, 4(4), 393-399. 
Alexander, R. M. (1985). Mechanics of posture and gait of some large dinosaurs. Zoological Journal of the Linnean Society, $83(1), 1-25$.

Arnold, P., Esteve-Altava, B., \& Fischer, M. S. (2017). Musculoskeletal networks reveal topological disparity in mammalian neck evolution. BMC Evolutionary Biology, 17(1), 251.

Arnold, P., Forterre, F., Lang, J., \& Fischer, M. S. (2016). Morphological disparity, conservatism, and integration in the canine lower cervical spine: Insights into mammalian neck function and regionalization. Mammalian Biology, 81(2), 153-162.

Asher, R. J., Lin, K. H., Kardjilov, N., \& Hautier, L. (2011). Variability and constraint in the mammalian vertebral column: Mammalian vertebral variability. Journal of Evolutionary Biology, 24(5), 1080-1090.

Baylac, M., \& Frieß, M. (2005). Fourier descriptors, procrustes superimposition, and data dimensionality: An example of cranial shape analysis in modern human populations. In D. E. Slice (Ed.), Modern morphometrics in physical anthropology (pp. 145-165). Boston: Springer.

Baylac, M., Villemant, C., \& Simbolotti, G. (2003). Combining geometric morphometrics with pattern recognition for the investigation of species complexes: Geometric morphometrics, pattern recognition and species complexes. Biological Journal of the Linnean Society, 80(1), 89-98.

Blender Foundation (2003-2018). Blender. Stichting Blender Foundation, Amsterdam,https://www.blender.org

Blomberg, S. P., Garland, T., \& Ives, A. R. (2003). Testing for phylogenetic signal in comparative data: Behavioural traits are more labile. Evolution, 57(4), 717-745.

Boas, J. E. V. (1929). Biologisch-anatomische Studien über den Hals der Vögel (pp. 1-127). Köbenhavn: A.F. Host \& Son.

Böhmer, C., Plateau, O., Cornette, R., \& Abourachid, A. (2019). Correlated evolution of neck length and leg length in birds. Royal Society open science, 6(5), 181588.

Böhmer, C., Rauhut, O. W. M., \& Wörheide, G. (2015). Correlation between Hox code and vertebral morphology in archosaurs. Proceedings of the Royal Society B: Biological Sciences, 282(1810), 20150077.

Bookstein, F. L. (1997). Morphometric tools for landmarks data: geometry and biology. Cambridge: Cambridge University Press.

Botton-Divet, L., Houssaye, A., Herrel, A., Fabre, A.-C., \& Cornette, R. (2015). Tools for quantitative form description; an evaluation of different software packages for semi-landmark analysis. PeerJ, 3, e1417.

Bout, R. G. (1997). Postures of the avian craniocervical column. Journal of Morphology, 231, 287-295.

Brocklehurst, R. J., Schachner, E. R., \& Sellers, W. I. (2018). Vertebral morphometrics and lung structure in non-avian dinosaurs. Royal Society Open Science, 5(10), 180983.

Cheverud, J. M. (1996). Developmental integration and the evolution of pleiotropy. American Zoologist, 36(1), 44-50.

Cobley, M. J., Rayfield, E. J., \& Barrett, P. M. (2013). Inter-vertebral flexibility of the ostrich neck: Implications for estimating sauropod neck flexibility. PLOS ONE, 8(8), e72187.

Cordeiro-Estrela, P., Baylac, M., Denys, C., \& Marinho-Filho, J. (2006). Interspecific patterns of skull variation between sympatric Brazillian vesper mice: Geometric morphometrics assessment. Journal of Mammalogy, 87(6), 1270-1279.

Cornette, R., Baylac, M., Souter, T., \& Herrel, A. (2013). Does shape co-variation between the skull and the mandible have functional consequences? A 3D approach for a 3D problem. Journal of Anatomy, 223(4), 329-336.

Daeschler, E. B., Shubin, N. H., \& Jenkins, F. A. (2006). A Devonian tetrapod-like fish and the evolution of the tetrapod body plan. Nature, 440(7085), 757-763.
Dasgupta, A., \& Raftery, A. E. (1998). Detecting features in spatial point processes with clutter via model-based clustering. Journal of the American statistical Association, 93, 294-302.

de Azevedo, T. P., Witten, P. E., Huysseune, A., Bensimon-Brito, A., Winkler, C., To, T. T., et al. (2012). Interrelationship and modularity of notochord and somites: A comparative view on zebrafish and chicken vertebral body development: Modularity of notochord and somites in zebrafish and chicken. Journal of Applied Ichthyology, 28(3), 316-319.

Diaconis, P., \& Freedman, D. (1984). Asymptotics of graphical projection pursuit. The Annals of Statistics, 12(3), 793-815.

Dilger, W. C. (2010). The comparative ethology of the African Parrot Genus Agapornis. Zeitschrift Für Tierpsychologie, 17(6), 649-685.

Ericsson, R., Knight, R., \& Johanson, Z. (2013). Evolution and development of the vertebrate neck. Journal of Anatomy, 222(1), $67-78$.

Everitt, B., \& Dunn, G. (2001). Applied multivariate data analysis (2nd ed.). Chichester: Wiley.

Fraley, C., \& Raftery, A. E. (1998). How many clusters? Which clustering meSthod? Answers via model-based cluster analysis. The Computer Journal, 41(8), 578-588.

Fraley, C., \& Raftery, A. E. (2012). mclust Version 4 for R: Normal mixture modeling for model-based clustering, classification, and density estimation. Department of Statistics, University of Washington, Technical Report no. 597.

Galis, F. (1999). Why do almost all mammals have seven cervical vertebrae? Developmental constraints, Hox genes, and cancer. Journal of Experimental Zoology, 285(1), 19-26.

Graf, W., Waele, C. D., \& Vidal, P. P. (1994). Functional anatomy of the head-neck movement system of quadrupedal and bipedal mammals. Journal of Anatomy, 186, 55-74.

Guinard, G. (2012). Evolutionary concepts meet the neck of penguins (Aves: Sphenisciformes), towards a "survival strategy" for evodevo. Theory in Biosciences, 131(4), 231-242.

Guinard, G., \& Marchand, D. (2010). Modularity and Complete Natural Homeoses in Cervical Vertebrae of Extant and Extinct Penguins (Aves: Sphenisciformes). Evolutionary Biology, 37(4), 210-226.

Guinard, G., Marchand, D., Courant, F., Gauthier-Clerc, M., \& Le Bohec, C. (2010). Morphology, ontogenesis and mechanics of cervical vertebrae in four species of penguins (Aves: Spheniscidae). Polar Biology, 33(6), 807-822.

Gunz, P., Mitteroecker, P., \& Bookstein, F. L. (2005). Semilandmarks in three dimensions. In D. E. Slice (Ed.), Modern morphometrics in physical anthropology (pp. 73-98). Boston: Springer.

Hackett, S. J., Kimball, R. T., Reddy, S., Bowie, R. C. K., Braun, E. L., Braun, M. J., et al. (2008). A Phylogenomic study of birds reveals their evolutionary history. Science, 320(5884), 1763-1768.

Hallgrímsson, B., Jamniczky, H., Young, N. M., Rolian, C., Parsons, T. E., Boughner, J. C., et al. (2009). Deciphering the palimpsest: Studying the relationship between morphological integration and phenotypic covariation. Evolutionary Biology, 36(4), 355-376.

Harmon, L. J., Weir, J. T., Brock, C. D., Glor, R. E., \& Challenger, W. (2008). GEIGER: Investigating evolutionary radiations. Bioinformatics, 24(1), 129-131.

Hautier, L., Weisbecker, V., Sanchez-Villagra, M. R., Goswami, A., \& Asher, R. J. (2010). Skeletal development in sloths and the evolution of mammalian vertebral patterning. Proceedings of the National Academy of Sciences, 107(44), 18903-18908.

Head, J. J., \& Polly, P. D. (2015). Evolution of the snake body form reveals homoplasy in amniote Hoxgene function. Nature, 520, 86-89.

Heidweiller, J., Van Der Leeuw, A. H. J., \& Zweers, G. A. (1992). Cervical kinematics during drinking in developing chickens. Journal of Experimental Zoology, 262(2), 135-153. 
Jetz, W., Thomas, G. H., Joy, J. B., Hartmann, K., \& Mooers, A. O. (2012). The global diversity of birds in space and time. Nature, 491(7424), 444-448.

Johnson, S. E., \& Shapiro, L. J. (1998). Positional behavior and vertebral morphology in atelines and cebines. American Journal of Physical Anthropology, 105, 333-354.

Jones, K. E., Angielczyk, K., \& Pierce, S. (2019). Vertebral regionalization facilitates functional diversification of the mammalian axial skeleton. The FASEB Journal, 33, 613.

Jones, K. E., Benitez, L., Angielczyk, K. D., \& Pierce, S. E. (2018). Adaptation and constraint in the evolution of the mammalian backbone. BMC Evolutionary Biology, 18(1), 172.

Long, J. H., Pabst, D. A., Shepherd, W. R., \& Mclellan, W. A. (1997). Locomotor desing of dolphin vertebral columns: bending mechanics and morphology of Dolphinus delphis. The Journal of Experimental Biology, 200, 65-81.

Kambic, R. E., Biewener, A. A., \& Pierce, S. E. (2017). Experimental determination of three-dimensional cervical joint mobility in the avian neck. Frontiers in Zoology, 14(1), 37.

Kembel, S. W., Cowan, P. D., Helmus, M. R., Cornwell, W. K., Morlon, H., Ackerly, D. D., et al. (2010). Picante: R tools for integrating phylogenies and ecology. Bioinformatics, 26(11), 1463-1464.

Klingenberg, C. P. (2008). Morphological Integration and Developmental Modularity. Annual Review of Ecology, Evolution, and Systematics, 39(1), 115-132.

Klingenberg, C. P. (2010). Evolution and development of shape: integrating quantitative approaches. Nature Reviews Genetics, 11, $623-635$

Klingenberg, C. P. (2014). Studying morphological integration and modularity at multiple levels: Concepts and analysis. Philosophical Transactions of the Royal Society B: Biological Sciences, 369(1649), 20130249-20130249.

Klingenberg, C. P., \& Marugán-Lobón, J. (2013). Evolutionary covariation in geometric morphometric data: Analyzing integration, modularity, and allometry in a phylogenetic context. Systematic Biology, 62(4), 591-610.

Krings, M., Nyakatura, J. A., Boumans, M. L. L. M., Fischer, M. S., \& Wagner, H. (2017). Barn owls maximize head rotations by a combination of yawing and rolling in functionally diverse regions of the neck. Journal of Anatomy, 231(1), 12-22.

Krings, M., Nyakatura, J. A., Fischer, M. S., \& Wagner, H. (2014). The cervical spine of the american barn owl (Tyto furcata pratincola): I. Anatomy of the vertebrae and regionalization in their S-shaped arrangement. PLoS ONE, 9(3), e91653.

Muller, J., Scheyer, T. M., Head, J. J., Barrett, P. M., Werneburg, I., Ericson, P. G. P., et al. (2010). Homeotic effects, somitogenesis and the evolution of vertebral numbers in recent and fossil amniotes. Proceedings of the National Academy of Sciences, 107(5), 2118-2123.

Oliver, J. D., Jones, K. E., Hautier, L., Loughry, W. J., \& Pierce, S. E. (2016). Vertebral bending mechanics and xenarthrous morphology in the nine-banded armadillo (Dasypus novemcinctus). The Journal of Experimental Biology, 219(19), 2991-3002.

Olson, E. C., \& Miller, R. L. (1958). Morphological integration. Chicago: University of Chicago Press.

Paradis, E., Claude, J., \& Strimmer, K. (2004). APE: Analyses of phylogenetics and evolution in R language. Bioinformatics, 20(2), 289-290.

Pierce, S. E., Ahlberg, P. E., Hutchinson, J. R., Molnar, J. L., Sanchez, S., Tafforeau, P., et al. (2013). Vertebral architecture in the earliest stem tetrapods. Nature, 494(7436), 226-229.
Polly, P. D., Head, J. J., \& Cohn, M. J. (2001). Testing modularity and dissociation: the evolution of regional proportions in snakes. In M. L. Zelditch (Ed.), Beyond heterochrony: The evolution of development (pp. 305-335). New York: Wiley.

R Core Team. (2017). R: A language and environment for statistical computing. Retrieved April 2, 2018, from https://www.R-proje ct.org/.

Randau, M., Cuff, A. R., Hutchinson, J. R., Pierce, S. E., \& Goswami, A. (2017). Regional differentiation of felid vertebral column evolution: A study of 3D shape trajectories. Organisms Diversity \& Evolution, 17(1), 305-319.

Revell, L. J. (2012). phytools: An R package for phylogenetic comparative biology (and other things): Phytools: R package. Methods in Ecology and Evolution, 3(2), 217-223.

Riedl, R. (1978). Order in living organisms: a systems analysis of evolution. New York: Wiley.

Rohlf, F. J., \& Slice, D. (1990). Extensions of the procrustes method for the optimal superimposition of landmarks. Systematic Zoology, 39(1), 40.

Romer, A. S. (1950). The vertebrate body. Philadelphia: W. B. Saunders Company.

Shapiro, L. J., \& Kemp, A. D. (2019). Functional and developmental influences on intraspecific variation in catarrhine vertebrae. American Journal of Physical Anthropology, 168(1), 131-144.

Schlager, S. (2013). Morpho: Calculations and visualisations related to geometric morphometrics. Retrieved April 2, 2018, from https ://sourceforge.net/projects/morpho-rpackage/.

Stevens, K. A., \& Parrish, J. M. (1999). Neck Posture and feeding habits of two jurassic sauropod dinosaurs. Science, 284(5415), 798-800.

Tambussi, C. P., de Mendoza, R., Degrange, F. J., \& Picasso, M. B. (2012). Flexibility along the neck of the neogene terror bird Andalgalornis steulleti (Aves Phorusrhacidae). PLoS ONE, 7(5), e37701.

van der Leeuw, H. J., Bout, R. G., \& Zweers, G. A. (2001). Control of the cranio-cervical system during feeding in birds. American Zoologist, 41, 1352-1363.

Villamil, C. I. (2018). Phenotypic integration of the cervical vertebrae in the Hominoidea (Primates): Cervical vertebrae integration in Apes. Evolution, 72(3), 490-517.

Virchow, H. (1910). Über die Bewegungsmöglichkeiten an der Wirbelsäule von Spheniscus. Sitzungsberichte der Gesellschaft Naturforschender Freunde zu Berlin. 1: 4-19.

Werneburg, I., Wilson, L. A. B., Parr, W. C. H., \& Joyce, W. G. (2015). Evolution of neck vertebral shape and neck retraction at the transition to modern turtles: An integrated geometric morphometric approach. Systematic Biology, 64(2), 187-204.

West-Eberhard, M. J. (2003). Developmental plasticity and evolution. Oxford: Oxford University Press.

Wickham, H. (2015). ggplot2: Elegant graphics for data analysis. Berlin: Springer.

Wiley, D. F., Amenta, N., Alcantara, D. A., Ghosh, D., Kil, Y. J., Delson, E., et al. (2005). Evolutionary Morphing. VIS 05 IEEE Visualization. doi: 10.1109/VISUAL.2005.1532826

Wilkinson, D. M., \& Ruxton, G. D. (2012). Understanding selection for long necks in different taxa. Biological Reviews, 87(3), 616-630.

Zweers, G. A., Vanden Berge, J. C., \& Koppendraier, R. (1987). Avian cranio-cervical systems. Part I: Anatomy of the cervical column in the chicken (Gallus gallus L.). Acta Morphologica NeerlandoScandinavica, 25, 131-155. 